Meta

Journal des traducteurs

Translators' Journal

\title{
Le « salto mortale de la déverbalisation »
}

\section{Jean-René Ladmiral}

Volume 50, numéro 2, avril 2005

Processus et cheminements en traduction et interprétation

Processes and Pathways in Translation and Interpretation

URI : https://id.erudit.org/iderudit/010994ar

DOI : https://doi.org/10.7202/010994ar

Aller au sommaire du numéro

Éditeur(s)

Les Presses de l'Université de Montréal

ISSN

0026-0452 (imprimé)

1492-1421 (numérique)

Découvrir la revue

Citer cet article

Ladmiral, J.-R. (2005). Le « salto mortale de la déverbalisation ». Meta, 50(2), 473-487. https://doi.org/10.7202/010994ar

\section{Résumé de l'article}

Le processus de la traduction n'obéit pas au schéma linéaire d'une séquence de transformations linguistiques présupposant qu'existent d'une langue à l'autre des " axes paraphrastiques » - ainsi qu'aimeraient le penser bien des linguistes et comme s'efforce nécessairement de l'opérationnaliser la traduction automatique (T.A.). Le plus souvent, au contraire, la traduction se caractérise par la discontinuité : le passage du texte-source (To) au texte-cible (Tt) implique un saut (saltus). Il se produit donc un processus de déverbalisation entre le texte original qui n'est " déjà plus " là et sa traduction qui n'est " pas encore ». Mais le concept de déverbalisation fait problème. Il parait évident en effet que le sens ne saurait exister sans un support - dont la nature reste à définir. Toujours est-il qu'en attendant les acquis scientifiques à venir d'une traductologie inductive, relevant des sciences cognitives, il y aura lieu de penser les processus de la traduction dans les termes d'une traductologie productive.
Ce document est protégé par la loi sur le droit d'auteur. L'utilisation des services d’Érudit (y compris la reproduction) est assujettie à sa politique d'utilisation que vous pouvez consulter en ligne.

https://apropos.erudit.org/fr/usagers/politique-dutilisation/ 


\title{
Le «salto mortale de la déverbalisation»
}

\author{
JEAN-RENÉ LADMIRAL \\ Université de Paris-X-Nanterre, Paris, France
}

\begin{abstract}
RÉSUMÉ
Le processus de la traduction n'obéit pas au schéma linéaire d'une séquence de transformations linguistiques présupposant qu'existent d'une langue à l'autre des «axes paraphrastiques »-ainsi qu'aimeraient le penser bien des linguistes et comme s'efforce nécessairement de l'opérationnaliser la traduction automatique (T.A.). Le plus souvent, au contraire, la traduction se caractérise par la discontinuité: le passage du texte-source (To) au texte-cible (Tt) implique un saut (saltus). II se produit donc un processus de déverbalisation entre le texte original qui n'est «déjà plus » là et sa traduction qui n'est «pas encore». Mais le concept de déverbalisation fait problème. II paraît évident en effet que le sens ne saurait exister sans un support - dont la nature reste à définir. Toujours est-il qu'en attendant les acquis scientifiques à venir d'une traductologie inductive, relevant des sciences cognitives, il y aura lieu de penser les processus de la traduction dans les termes d'une traductologie productive.
\end{abstract}

\begin{abstract}
Contrary to what many linguists are led to think, and to what machine translation is bound to assume in its operations, the translation process does not follow a linear sequence of language transformations, presupposing phrasal equivalences from one language into another. The translation is characterized, most of the time, by discontinuity the transfer from the source language into the target language is operated by a rupture (saltus). A procedure of "deverbalization" (un-wording) occurs between the source text which is "no longer there" and the target text which is "not yet here." But the concept of "deverbalization" remains problematic, because meaning cannot exist without a medium, the nature of which is still to be determined. The prospective developments of cognitive sciences will have to afford the basis of an inductive translatology, meanwhile we have to think out the process of translation in the frame of a productive translatology.
\end{abstract}

\section{MOTS-CLÉS/KEYWORDS}

déverbalisation, psychologie, épistémologie, science

au général Massu

Parmi les processus que met en œuvre la traduction, il se passe quelque chose qu'il y aura lieu d'appeler une « déverbalisation». Cela dit, ce concept de déverbalisation fait problème. - À d'aucuns, il «donne des boutons»! comme à mon collègue et ami Jacky Martin (voir Hewson et Martin 1991). Pour un littéraire, et particulièrement pour un littéraire de haut vol comme lui, on comprend que ce concept soit irrecevable dans la mesure où ce qu'il paraît mettre en cause, c'est la texture langagière qui fait la substance même de la littérature, tant il est vrai qu'une œuvre littéraire est une œuvre d'art qui ne prend pour matériau qu'elle travaille rien d'autre que le langage, dirai-je pour faire écho à une formule devenue classique de Wolfgang Kayser (das sprachliche Kunstwerk). Pour un philosophe - comme moi ou plutôt, en l'occurrence, 
comme d'autres -, il semble qu'il n'en aille pas autrement et qu'on en revienne là tout simplement à la problématique traditionnelle des rapports entre le langage et la pensée. En clair : existe-t-il une pensée avant le langage? existe-t-il une pensée sans le langage? À quoi les Modernes inclinent à répondre: non! par opposition à la pensée classique, taxée d'idéalisme.

Plus concrètement, une des réserves qu'appellera le concept de déverbalisation tient au fait qu'il est apparu dans le cadre de la «théorie interprétative de la traduction » (T.I.T.) qu'ont développée Danica Seleskovitch et Marianne Lederer, au sein de de l'École Supérieure d'Interprètes et de Traducteurs (É.S.I.T.) de l'Université de ParisIII (voir Seleskovitch et Lederer 1984). Or il est indéniable que c'est d'abord la traduction professionnelle que la T.I.T. a prise pour champ d'étude et que, du coup, s'en trouvait écartée a priori la traduction littéraire, et minimisée l'importance accordée aux signifiants du langage. S'agissant en l'espèce d'un aspect tout à fait essentiel du problème, on conçoit que pour ceux qui se sont assigné la traduction des textes littéraires comme «terrain» de leur recherche traductologique, voire de leur pratique traductive, comme c'est le cas d'un grand nombre de nos collègues universitaires au sein des départements de langues vivantes (ou anciennes), il y ait là matière aux plus grandes réticences. Entre temps, il est vrai qu'il a été décidé à l'É.S.I.T. de ne plus exclure la traduction littéraire et de la réintégrer dans le champ des recherches menées dans la mouvance de la T.I.T. ${ }^{1}$

Cela dit, la question n'est pas celle du champ d'application spécifique de telle ou telle approche traductologique. Par exemple: je m'inscris résolument en faux contre l'idée avancée par Antoine Berman que la «traduction des œuvres» (et donc notamment la traduction littéraire) relèverait d'une théorie de la traduction littéraliste ou «sourcière» (pour reprendre un de mes propres concepts), alors que pour la traduction professionnelle (c'est-à-dire, si l'on en croit le même Berman, la traduction de la «parole creuse»!) on pourrait se contenter d'une théorie «cibliste» (Ladmiral 1986). Pas plus, par exemple, que la controverse qui nous a opposés (et aussi, en un sens, rapprochés), Henri Meschonnic et moi-même, ait tenu (comme il a semblé vouloir le croire) au fait qu'il est traducteur de la Bible, où il décide de ne voir que des «textes poétiques ", alors que je me suis adonné quant à moi à la traduction des grands textes de la philosophie allemande (voir Ladmiral et Meschonnic 1981). Si la théorie de la traduction a quelque chance d'atteindre à la vérité (s'il lui revient un Wahrheitsgehalt comme disent les philosophes allemands), elle doit rendre compte de l'ensemble. Surtout: ce n'est pas sur le plan du «langage-objet» des différents domaines de la pratique traduisante que le problème se pose, mais sur le plan du métalangage épistémologique auquel renvoie la théorie traductologique.

Sur le plan des faits, de quoi s'agit-il? Au départ, il y a une évidence de bon sens, incontournable. Dès lors que traduire un texte $(\mathrm{T})$, c'est le faire passer d'une langue (Lx) à une autre (Ly), d'une langue-source (Lo) à une langue-cible (Lt), il faut supposer - on ne peut pas ne pas supposer qu'il y a eu un décrochement des signifiants linguistiques, c'est-à-dire d'abord un décrochement des signifiants de la languesource. Et par quel miracle s'imaginerait-on que ce texte se trouverait d'emblée réincarné dans les signifiants de la langue-cible? dans un corps de signifiants tout autres! Entre ces deux moments, il y a nécessairement une interface qui les articule l'un à l'autre, et que je me plais à appeler un no man's langue. C'est là très précisément en quoi réside ladite déverbalisation, tout simplement. 
Ainsi y a-t-il lieu de s'étonner que ce malheureux concept déclenche une telle levée de boucliers (mirari vos...). Encore une fois, dans l'opposition frontale d'un Jacky Martin (par exemple) à l'idée même de déverbalisation, ce qui est en cause, ce n'est pas tant une différence de «terrains» traductologiques qu'une divergence de nature épistémologique, à l'arrière-plan de laquelle on sera à vrai dire fondé à soupçonner la surdétermination de motivations d'ordre psycho-sociologique plus ou moins conscientes. En effet, ce qui insupporte des universitaires qui s'occupent de traduction, qu'ils soient littéraires ou même éventuellement linguistes, c'est aussi le côté dogmatique des positions de Danica Seleskovitch et la façon polémique qu'elle avait de les défendre.

Mais cette virulence était elle-même en grande partie une réaction, qui à son tour mettait en jeu des ressorts d'ordre psycho-relationnel et socio-institutionnel, venant interférer là encore avec les considérations proprement théoriques. Pour donner à la traduction la place qui lui revient et lui conférer (enfin!) un statut universitaire, il a fallu que Seleskovitch affronte en son temps bien des résistances émanant d'une «vieille garde» universitaire élitiste et traditionnaliste qui, au pire, refusait de voir les études supérieures commises aux tâches «ancillaires» de la traduction et qui, au mieux, voulait ne voir dans cette dernière que le je-ne-sais-quoi inspiré de l'artiste, une prouesse littéraire qui ne s'enseigne pas, pour autant qu'on n'en saurait révéler «les coulisses de l'exploit». On conçoit qu'une personnalité énergique qui s'était donné pour tâche de rénover la formation universitaire des traducteurs (et des interprètes) en soit venue, dans ce contexte, à céder aux tentations de l'agacement, et même parfois de l'arrogance. S'agissant de Danica Seleskovich, je me risquerai à parodier un mot historique bien connu: Mère, gardez-vous à droite! (de la littérature); Mère, gardez-vous à gauche! (de la linguistique)... Cela dit, elle n'a pas été non plus la seule; et d'autres, en d'autres lieux, ont dû livrer le même combat.

Au reste, au-delà des personnalités que je viens de citer nommément d'un côté ou de l'autre, ce qui est en cause, c'est une problématique plus générale, concernant les processus en traduction et corollairement les problèmes institutionnels, notamment en matière de formation. En l'occurrence, l'attitude des individus (que nous sommes, tous) ne fait qu'illustrer ici (et ailleurs) la logique des «fronts scientifiques » - et didactiques - qui traverse nécessairement la recherche et l'enseignement; tant il est vrai qu' «il n'y a pas de genèse intellectuelle sans genèse sociale» (Lourau 1978). Il reste que les appartenances socio-professionnelles tendent à surdéterminer les attitudes intellectuelles de ces individus que sont encore les chercheurs. Sur le plan psychoindividuel, il se produit ce que j'appellerai une identification disciplinaire, en vertu de laquelle on est porté à «se cramponner» à sa méthodologie, c'est-à-dire à la méthodologie dominante au sein du cadre disciplinaire dans lequel on se reconnaît.

Ainsi, par exemple, les spécialistes de didactique des langues n’ont-ils longtemps voulu voir que la dimension linguistique des problèmes que pose l'apprentissage d'une langue seconde et cela reste encore assez largement le cas, en raison tout simplement du fait que ce sont très majoritairement des linguistes... En sorte que les déterminations psychologiques et sociologiques, mais aussi culturelles et même politiques de la question n'ont été que très insuffisamment prises en compte - en dépit du travail de pionnier (de "pionnière»?) qu'avait en son temps entrepris une Danielle Bailly par exemple (voir Bailly 1984). Et, au bout du compte, les choses ne sont pas fondamentalement différentes dans le domaine de la traduction: encore 
maintenant, l'essentiel de la littérature traductologique est d'obédience linguistique et ressortit à ce que j'ai appelé la traductologie descriptive (Ladmiral 1987b).

Mais si, au lieu de s'en tenir à une telle approche linguistique et «descriptive» prenant pour objet l'après-coup d'une traduction déjà faite, on va du côté de ce que j'ai appelé une traductologie productive, qui prend en compte les processus en traduction et s'efforce d'anticiper la traduction à faire (ou «se faisant», comme dirait Bergson), si donc on se place in medias res, alors on se retrouvera nécessairement confronté à ce moment de l'entre-deux qui implique la déverbalisation, ainsi qu'il a été indiqué. Il m’apparaît en effet que, comme j’y ai insisté déjà à plusieurs reprises (voir Ladmiral 1997), la traduction est foncièrement un phénomène binaire. Le vécu du traducteur est radicalement clivé, entre ces deux phases:

- $\quad$ une phase (I) de lecture-interprétation;

- $\quad$ une phase (II) de réexpression (rewording), c'est-à-dire de réécriture, dès lors qu'il s'agit de traduction (stricto sensu), portant donc sur des textes écrits.

Je veux bien qu'on dise que la traduction est une opération triangulaire (comme on l'enseigne à l'É.S.I.T.), au sens où le processus de la traduction n'est pas un processus linéaire, c'est-à-dire qu'on ne passe pas directement du texte-source (To) au texte-cible de sa traduction $(\mathrm{Tt})$, dans la mesure où le moment de la déverbalisation est à l'articulation des deux phases qui viennent d'être indiquées. Mais, à mon sens, il n'y a pas lieu de thématiser ce moment médian (et médiateur) comme une phase spécifique: tout au plus est-ce là ce qu'on pourrait appeler une «interphase». En somme, il y aurait trois moments définissant deux phases - et si je voulais jargonner, en métaphorisant le langage mathématique (quitte à prêter le flanc à des critiques «genre Sokal»), je dirai: trois points délimitant deux segments orientés ou vectoriels. Car, quand on traduit, c'est dans le même mouvement de la phase (I) qu'on va du moment de la lecture au moment de la déverbalisation, pour autant qu'on peut faire fond sur la disponibilité constante du texte-source (To), auquel il est toujours possible de revenir; et c'est encore dans le même mouvement de la phase (II) qu'on va du moment de la déverbalisation au moment de la réécriture du texte-cible $(\mathrm{Tt})$.

Mais sans doute est-on fondé à analyser le processus de l'interprétation consécutive de façon légèrement différente. Les conditions de l'exercice ne sont en effet plus tout à fait les mêmes que celles dans lesquelles se réalise le processus de la traduction (stricto sensu) : comme on sait, elles différent sur deux points essentiels. D'une part, on est là dans la temporalité volatile de l'oral et la matérialité écrite du texte-source n'est plus constamment accessible (verba volant). D'autre part, le message-source qu'émet l'orateur, et qu'il s'agira d' «interpréter», se trouvera fractionné en séquences de l'ordre de cinq à dix minutes, de sorte qu'elles ne sont pas susceptibles d'être engrammées en mémoire immédiate, et «traduites» instantanément, comme ce peut être le cas en interprétation simultanée. Dès lors se pose le problème du stockage des informations: du coup, le moment intermédiaire correspondant à la déverbalisation va s'autonomiser ou plutôt ce sera le moment médiateur du stockage qui va être matérialisé par la prise de notes. Il y a là une rubrique bien connue dans le cadre de nos écoles de traduction (la TPN). S'agissant du point qui m'occupe ici, disons que le moment médiateur de l'«interphase» articulant les deux phases de la traduction (écrite) donne lieu en l'espèce à une phase intermédiaire à part entière intercalant un relais écrit (ou peut-être quasi-écrit, ou plutôt graphique) au sein de la «traduction 
orale» qui, donc, devient un processus (un procédé) de traduction effectivement «triangulaire» ou ternaire, comportant trois phases distinctes.

Il en va de même pour une pratique particulière très proche de l'interprétation consécutive pour laquelle je ne sais pas trop quelle appellation adopter, hésitant entre interprétation mini-consécutive, "semi-consécutive» ou quasi-simultanée... J'entends par là une procédure consistant à proposer l'équivalence traductive de séquences extrêmement brèves du message-source, de moins d'une minute, dès lors que l'orateur accepte (et se montre capable) de jouer ce jeu.

Cette façon de faire est certes un peu éprouvante pour l'orateur, mais elle présente pour lui le double intérêt de lui laisser un droit de regard sur l'interprétation qui est donnée de ses propos (quand il connaît plus ou moins l'autre langue) et de lui permettre de «rectifier le tir» ou, plus souvent, de rebondir sur le message-cible de l'interprétation et de revenir sur tel ou tel point pour l'approfondir. Ainsi lui est-il accordé une certaine marge de créativité verbale et intellectuelle. Sans parler du fait que c'est en outre une façon de ménager une certaine "respiration » dans la conférence, aussi bien pour l'orateur que pour ses auditeurs, permettant à l'un comme aux autres de «souffler» et de prendre le temps de la réflexion. Il y a là un effet de redondance interlinguistique qui est de nature à rendre les choses plus légères; et ce peut être la bonne façon de procéder quand le message-source est de haute volée théorique et d'une grande densité conceptuelle.

À quoi vient s'ajouter un autre avantage pour le public qui, sur la base de ces empans de traduction très courts, dispose des deux versions, en sorte qu'il peut luimême les comparer et contrôler en partie l'interprétation proposée. Cela suppose évidemment que ce public ait une relative compétence dans la langue-source; mais cela arrive assez souvent dans le domaine de la communication «scientifique» (lato sensu), d'autant que les langues concernées sont en l'occurrence souvent des langues dans lesquelles ont été rédigés les travaux auxquels il est fait référence (et qu'ont lus les membres du public), comme l'anglais, le français ou l'allemand, par exemple. Pour toutes ces raisons, on comprend que certains préfèrent cette procédure du «haché menu» (pour reprendre une expression de mon collègue Dominique Petit). Mais il est bien clair aussi que c'est très incommode pour l'interprète. Cela lui impose la tension d'un grand effort de concentration et de travail de la mémoire; et puis ce n'est pas sans risque pour lui: mutatis mutandis, il est quasiment dans la position d'un dompteur dans un cirque, qui a l'impression que le public est venu en espérant un peu que ce sera (enfin) la séance où il sera dévoré par ses lions! Il m’est arrivé assez souvent de pratiquer cette méthode. C'est ainsi par exemple que je me souviens encore de l'interprétation (allemand-français) que j'ai dû récemment fournir de Reinhard Koselleck au Collège de France, devant un parterre de sommités connaissant souvent bien l'allemand, et j'ai encore en tête le fameux sourire énigmatique (gourmand?) du regretté Pierre Bourdieu...

Dans le cadre de cette dernière procédure de traduction orale, le stockage intermédiaire des informations n'est plus assuré par la prise de notes et matérialisé sur du papier: il est d'ordre psycho-mémoriel. Le moment de la déverbalisation est devenu une phase sui generis de mémorisation. Ce qui nous donne le schéma suivant du processus de «traduction» (orale):

1) il s'agit d'abord d' «entendre» le message (To), c'est-à-dire de l'« ouïr» et surtout de le comprendre (voir lecture-interprétation); 
2) puis de le «retenir», c'est-à-dire d'en mémoriser le contenu (déverbalisation) pendant le laps de temps qu'occupe l'empan de parole qui revient à l'orateur qu'on a à «traduire», ce qui implique effectivement une «conceptualisation» et le passage de la mémoire immédiate à la mémoire sémantique (voir Ladmiral 2002a: 36);

3) pour enfin être en mesure de le «re-dire», de le réexprimer dans la langue-cible (Lt).

On pourra penser aussi à d'autres variantes du processus de traduction comme, par exemple, dans le cas des groupes bilingues (ou plurilingues), où la médiation interlinguistique est assurée «sur le tas» par les individus membres du groupe qui s'en sentent la compétence et le goût. Dans ce cadre-là, il n'est procédé à «la traduction» (orale) que de façon informelle et subjective, c'est-à-dire de manière sélective et souvent orientée, et surtout au coup par coup. D’une façon générale, les processus de traduction interfèrent là avec la logique psycho-sociologique de la dynamique de groupe, ce qui pourra donner matière à une pratique de l'animation de communication interculturelle ${ }^{2}$.

Quoi qu'il en soit de savoir si la communication traductive est binaire ou triangulaire, il ressort bien de l'excursus où viennent d'être esquissées plusieurs modalités d'«interprétation consécutive» que les processus de traduction (et, plus généralement, de «communication interlinguistique») mettent effectivement en ouvre un moment médiateur de déverbalisation, qui pourra soit prendre la forme proprement matérielle du papier dans la prise de notes, soit en rester sur le plan de l'expérience psychologique, voire d'un pratique psycho-sociologique. Autant de processus différents de «traduction orale». Dans le cas de la traduction proprement dite - s'agissant de textes écrits, ainsi qu'il a été rappelé -, cette interphase est de nature psychologique ou, si l'on veut, «mentale». Plus précisément, pour reprendre une formule que j'affectionne, le message (le contenu du message) passe du niveau verbo-linguistique à un niveau psycho-cognitif. C'est un point sur lequel on insiste à juste titre à l'É.S.I.T. en parlant aussi de conceptualisation pour qualifier ce moment inter-médiaire de la déverbalisation. Cela dit, sur la dimension psychologique du problème, il convient de faire trois remarques.

D'abord, bien entendu, précisons-le tout de suite: la psychologie dont il est question en l'occurrence est une psychologie pour non-psychologues, comme je l'ai déjà marqué en quelques occasions (voir Ladmiral 2004: 39). Sans doute pourrat-on parler à ce propos d'une "psychologie linguistique», conformément à une formule de Jacques Pohl. Surtout, il s'agit de «psychologie de l'intelligence» (comme on disait encore aux temps où j'étais étudiant «en psycho») qui, contrairement à une mode qui n'est que trop répandue actuellement dans le milieu des sciences humaines, ne prétend pas, en invoquant le concept-shibboleth de cognition, se créditer du prestige épistémologique des sciences cognitives, lequel reste encore très largement programmatique pour les disciplines qui nous concernent ${ }^{3}$. Enfin, pour en revenir au concept de déverbalisation lui-même, j'ai longtemps cru et dit parfois (peut-être même écrit!) que ce concept était emprunté aux psychologues jusqu'à ce que Danica Seleskovitch m'en inflige oralement un démenti formel et m'assure que c'était un concept original, créé dans la dynamique de la recherche traductologique qu'elle animait. Dont acte! comme quoi, la culture interdisciplinaire qu'on s'est donné la peine d'acquérir, et qu'on croit encore avoir, peut vous jouer des tours...

Mais si l'on est bien forcé d'admettre que le processus de la traduction en passe par le saltus de ce que j'ai appelé un «no man's langue», qui se situe entre le déjà-plus 
du message-source (To) et le pas-encore du message-cible ( $\mathrm{Tt}$ ), dirai-je en me permettant de pasticher mon bon maître Vladimir Jankélévitch. Alors comment se fait-il que d'aucuns se montrent radicalement «allergiques » à l'idée même de déverbalisation (voir sup.). Ainsi qu'il a été indiqué, cette surdité conceptuelle remonte en partie à des considérations d'ordre philosophique ou épistémologique. Dès lors qu'on a décroché de l'approche littéraire ou de la méthodologie linguistique, qui prennent pour objet des énoncés observables, il semble en effet qu'on veuille réactiver l'interdit béhaviouriste de tout «mentalisme» et que certains soient la proie d'une régression faisant une fixation (comme diraient les psychanalystes), pour ainsi dire «cramponnés» à leur méthodologie de départ. Il m’apparaît qu'il y a là ce que Heidegger appelle un «recul de la pensée».

Au reste, cet interdit méthodologique et épistémologique trouvera à se soutenir de considérations plus concrètes, relevant d'une philosophie du langage. Dans cet esprit, ce n'est pas seulement l'inconscient qui est "structuré comme un langage» (comme disait Jacques Lacan), c'est aussi la conscience et la pensée elle-même. Du coup, le concept de déverbalisation lui-même ne fait-il pas figure de contradictio in terminis? et ce que j'appelle le «salto mortale de la déverbalisation» semblerait en appeler à un état de grâce insaisissable, voué à une inassignable thaumaturgie du je-ne-sais-quoi, à une métaphysique de l'ineffable. Et voilà pourquoi votre fille est muette! (dirai-je pour citer Molière).

Cette double objection appelle une réponse globale. Que notre vie mentale soit fondamentalement conditionnée par notre rapport au langage, on n'en disconviendra pas; et quand la psychanalyse Françoise Dolto nous dit que, dès la prime enfance, l'être humain est ce qu'elle s'est plu à appeler un "parlêtre», nous en sommes aisément convaincus. Encore faudra-t-il faire la différence entre plusieurs niveaux de communication verbale, et de communication «non verbale» ou plutôt sans doute «para-verbale». Le concept de déverbalisation ne présuppose pas un moment de la vie mentale qui soit mutique entre deux moments de verbalité, mais bien plutôt que, dans cet entre-deux, il y a nécessairement un décrochement d'avec la forme achevée et conforme à la norme des deux langues «en contact» (Lo et Lt). Il paraît incontestable que toutes nos représentations et toute communication ne peuvent exister sans un «support». Mais ce support, ce ne sont pas nécessairement les signifiants d'une langue, d'une seule langue, ni surtout que ces signifiants soient organisés en un énoncé bien formé (ÉBF) conforme à la norme d'une langue naturelle donnée.

Cette problématique du support trouve à s'illustrer à l'occasion notamment des remarques qui ont été faites à propos de l'interprétation consécutive. Ainsi, la "prise de notes» n'y débouche-t-elle jamais sur du verbal «en forme», mais sur quelque chose où s'entremêlent le para-verbal, l'infra-verbal et le non-verbal, par exemple l'iconique. Et pourtant, c'est bien sur la base de ce support hétéroclite et schématique que l'interprète sera en mesure d'opérer une verbalisation complète, c'est-à-dire de produire en langue-cible un discours «bien formé » $(\mathrm{Tt})$ adéquat au contenu dont était porteur le message-source (To); et ce, lors même que ces notes seront totalement inutilisables par quelqu'un d'autre que celui qui les a "prises», et qu'elles auront même perdu tout leur sens pour ce dernier, ne fût-ce que quelques heures après sa prestation. Un autre exemple: quand nous improvisons une prise de parole en public - au sein d'un débat, à l'occasion de la discussion qui suit la communication d'un collègue dans le cadre d'un colloque scientifique par exemple, ou dans le 
cadre d'un affrontement politique et idéologique -, est-ce que nous avons rédigé notre intervention? Non, bien sûr: nous développons un discours structuré et argumenté à partir de quelques «gribouillis» que nous venons de griffonner sur un bout de papier, illisible en soi mais sans lequel nous serions «perdus».

En somme: la traduction d'un texte implique qu'en soit dégagé le «sens» (en donnant à ce concept un... sens très large), qui reste une sorte de nébuleuse dans la mesure où le "salto mortale de la déverbalisation" fait qu'il se trouve privé de son support normal que constituent les signifiants d'une langue naturelle (déjà plus Lo et pas encore Lt). Autrement dit, comme se plaisait à le répéter Daniel Moskowitz, on ne traduit pas des mots mais des idées (voir Ladmiral 2002: 220). Ces idées - que j'ai proposé d'appeler aussi des sémantèmes (Ladmiral 2002: 206) en donnant à ce terme vieilli le sens renouvelé d'un concept traductologique -, quel en sera le support «hétéroclite et schématique» (voir sup.? Qu'ils en restent au stade d'une espèce de «brouillon mental» ou qu'ils aillent jusqu'à prendre la forme matérielle d'une sorte de prise de notes plus ou moins rudimentaire, ce seront des items hétérogènes au nombre desquels pourront figurer des «signifiants» très divers:

- des mots isolés au sémantisme diffluent (des «mots-idées»), appartenant à l'une ou l'autre des deux langues en présence ou aux deux (Lt et/ou Lo), ou même à une autre langue (Lx) ou deux (Ly, Lz...);

- des abréviations;

- des opérateurs logiques, auxquels correspondent les symboles de la logique formelle et qu'on peut paraphraser en langue naturelle (si... alors..., ou, et, la négation, etc.);

- ce que j'appellerai des opérateurs binaires de clivage orienté, qui tendront souvent à prendre la valeur d' «opérateurs axiologiques», de marqueurs d'inspiration booléenne: droite/gauche, haut/bas, oui/non, bon/mauvais, je suis pour/je suis contre, passé/futur, etc.

- des images mentales (comme on disait au bon temps de l'associationnisme);

- des croquis et des esquisses; etc.

On se situe là sur un plan qui oscille entre le verbal et le protoverbal, le paraverbal et le non-verbal, par exemple l'iconique. S'agissant d'une telle nébuleuse sémantique, on pourra en chercher des éléments d'analyse du côté de la linguistique cognitive (voir Langacker, Montaguë, etc.). Mais, au sein des sciences cognitives, c'est plus profondément du côté de la psycholinguistique qu'il y aura lieu d'attendre des éclaircissements sur le plan de la réalité objective du fonctionnement mental. Cela dit, on est encore loin du compte. C'est pourquoi, en attendant, on ne doit pas s'interdire d'enfreindre le tabou épistémologique du «mentalisme». Au reste, plusieurs chercheurs n'ont pas craint depuis déjà quelques années de recourir aux données de l'introspection. Telle était, par exemple, la méthodologie empruntée par Hans Peter Krings pour décrire «ce qui se passe dans la tête des traducteurs» (Krings 1986).

Quant au concept de déverbalisation lui-même, je tiens à préciser que c'est un concept minimaliste et qu'il est purement phénoménologique. Il s'agit d'un simple étiquetage qui prend en compte la réalité d'un vécu qui est celui du traducteur. Pour ceux qui y ont recours, le terme de déverbalisation ne constitue pas un concept auquel serait attachée la valeur d'une explication scientifique. Il n'est pas question en l'occurrence de prétendre qu'on aurait ouvert la «boîte noire», dirai-je pour reprendre un concept de la psychologie béhaviouriste qui, avec Skinner, a été introduit en linguistique (black box), qu'on aurait «vu ce qu'il y a dedans»! qu'on saurait vraiment 
ce qui s'y passe et qu'on serait en mesure de l'expliquer. Sur cet aspect de la question, on ne peut dans l'état actuel de nos connaissances que continuer à faire une impasse épistémologique dans l'esprit de ce qu'un Werner Heisenberg a appelé la connaissance incomplète (voir Ladmiral 1971).

Mon propos va seulement servir à opérer ce que j'appellerai un déplacement de la ligne d'interdit épistémologique. Certes, on ne sait pas encore ce qui se passe vraiment dans le cerveau du traducteur. C'est l'objet de ce que j'ai appelé la traductologie inductive, c'est-à-dire d'une traductologie proprement scientifique qui se situe en aval de ce versant des sciences cognitives que constituent les neurosciences. Mais, dans la foulée de ce qui vient d'être dit et compte tenu des échéances de recherche en cours, il m'est apparu que ce ne pouvait être encore que la "traductologie de demain", voire d'après-demain ${ }^{4}$ ! - En revanche, il est possible de prendre connaissance de ce qui se passe dans la tête des traducteurs. C'est ce que s'est efforcé de faire un Hans Peter Krings de façon empirique, en ayant recours à une méthode introspective pour étudier les parcours de résolution de problèmes (problem-solving) suivis des différents sujets engagés dans un processus de traduction (voir sup.); et c'est aussi ce que j'ai fait moi-même ici en adoptant une approche phénoménologique, avec mon schéma binaire censé illustrer ledit salto mortale de la déverbalisation.

Plus généralement, c'est dans cet esprit que je me suis attaché à thématiser l'idée de traductologie productive - à la fois par défaut et par excès, pour ainsi dire. Par défaut: dans la mesure où la traductologie inductive est la traductologie de demain (ou d'après-demain) et où elle n'est donc évidemment pas encore en mesure de nous fournir un modèle général dont il serait possible de dégager aujourd'hui des «applications» susceptibles d'être mises en œuvre dans le cadre de la pratique traduisante, il conviendra de se forger une "morale par provision», comme disait Descartes. C'est-à-dire qu'on devra gérer le processus de la traduction au jour le jour à partir d'une «connaissance incomplète», en attendant un savoir objectif et plus assuré. C'est au demeurant ce qu'on ne cesse de faire dans la pratique en général, d'autant qu'il y a une clairvoyance propre à la pratique, qui lui est inhérente au niveau qui est le sien. Autrement dit: dans savoir-faire, il y a déjà savoir ${ }^{5}$. Freud n'en use pas autrement qui, en tant que médecin, voulait soulager la souffrance (morale) de ses patients, mais qui déplorait que n'ait pas existé de son temps une neurologie scientifique assez avancée pour lui fournir les solutions et les remèdes dont il avait besoin, en sorte qu'il a dû «bricoler» progressivement, au jour le jour, l'invention d'un savoir empirique qui lui a permis de développer une méthode thérapeutique, à laquelle il a donné le nom de psychanalyse...6

Il ne sera guère possible, dans le cadre limité de la présente étude, de définir «par excès» (comme on dit) la traductologie productive. Je me contenterai d'indiquer qu'il s'agira de mettre en place la discursivité d'une culture traductologique qui permettra d'éclairer la pratique, de désinhiber le sujet traduisant et de lui permettre, quand il est encore en formation (mais ne le sommes-nous pas toujours?), de prendre la mesure de son idiosyncrasie de traducteur. Il s'agira de développer une discursivité traductologique dont certains moments «cruciaux» pourront donner lieu à la formulation de concepts (mots) et principes (phrases) qui seront autant de théorèmes pour la traduction $^{7}$. La traductologie productive est une "praxéologie» (Handlungswissenschaft) dont la finalité est d'anticiper la traduction à faire (alors que la traductologie descriptive s'en tient à analyer a posteriori la traduction déjà faite, et le plus 
souvent par d'autres!). - Entre temps, il m'est apparu que la différence entre traductologie productive et traductologie inductive n'est pas seulement une affaire de calendrier de la recherche, mais qu'elle correspond à l'alternative épistémologique de deux approches théoriques distinctes que sous-tendent deux modalités cognitives respectivement spécifiques (Ladmiral 2001: 110-111).

Plus précisément, comme il vient d'être indiqué, sa fonction est d'apporter une aide au traducteur en difficulté. Ce n'est pas toujours le cas: il y a des plages entières de texte (To) que nous traduisons sans grande difficulté; et quand ça va tout seul, pour ainsi dire, point n'est besoin de traductologie ni de traductologues. Mais l'une des difficultés à laquelle on se trouve assez souvent confronté en traduisant est la nécessité de devoir "décrocher» complètement des signifiants de la langue-source, de la Lettre du texte-source, pour produire une bonne traduction, qui ne «sent » pas la traduction, qui est donc à la fois fidèle à l'Esprit du texte (To) et conforme aux ressources spécifiques de la langue-cible ( $\mathrm{Lt})$ : c'est ce que j'appelle la dissimilation (voir Ladmiral 2002a: 57, 198, 218, etc. et 1998: 149-150). En effet, certains textes «habitent» si bien la langue dans laquelle ils ont été écrits (Lo) qu'on a la plus grande peine à les en arracher et qu'on s'hypnotise sur les signifiants-source, si parfaitement adéquats, que le texte met en «œuvre»: on le lit, on le relit, on le re-relit, on le re-re-relit, on le re-re-re-relit... comme s'il allait miraculeusement sourdre d'entre les lignes de l'original, comme en filigrane, sa traduction! par effet de palimpseste fantasmatique. Il y a là un processus en traduction bien connu de ceux qui la pratiquent, avec corrélativement l'expérience frustrante d'une perte des moyens d'expression qui serait comme une sorte de "castration linguistique (voir Ladmiral 2002a: 25).

À la réflexion, ce qui vient d'être évoqué n'est qu'une illustration relativement marquante du processus de déverbalisation dans la traduction, qui fait l'objet de la présente étude. Et si j'ai cru bon d'y ajouter une «touche personnelle», en en faisant le «théorème du salto mortale de la déverbalisation» - qui m'a fourni ici la matière de mon titre -, c'est précisément pour y apporter un élément de dramatisation soulignant la tension psychologique qu'implique un tel travail mental de reformulation (rewording) quand il lui faut ainsi rompre toutes les amarres d'avec la forme de l'énoncé-source (voir Ladmiral 2002b: 132).

Cela dit, il est vrai aussi que tout n'est pas toujours aussi «tendu» et que le traducteur n'est pas toujours «en difficulté». Il arrive même qu'il semblerait qu'on pût traduire en restant en "structure de surface» et en opérant de simples transformations linguistiques, comme s'il y avait des «axes paraphrastiques » d'une langue à une autre. Cela n'est certainement pas la réalité (voir Ladmiral 2002a: 121-126). Mais l'idée qu'il y ait des équivalences traductives constantes d'une langue à une autre (Übersetzungsregularitäten) se trouve en partie "plausibilisée» du fait que la plupart des langues qui nous occupent pour ce qui est de la traduction sont des langues apparentées (indo-européennes), ayant notamment les mêmes «parties du discours», et que rapprochent tout un ensemble de cousinages culturels (au moins jusqu'à présent). En ce sens, il y aurait lieu peut-être d'amodier (un peu) la critique des « contrastivistes » que j'ai faite (Ladmiral 1987a:21) et de procéder parallèlement à une ébauche de rapprochement entre traductologie productive et traductologie descriptive. Il reste toutefois que ces équivalences (ou concordances) inter-linguistiques, qui sembleraient aller à identifier parfois la traduction à un pur et simple transcodage, ne 
sont pas la règle; et quand la chose apparaît possible, il reste que ces équivalences linguistiques sont pour ainsi dire enchâssées dans le cadre méta-communicationnel d'une pragmatique de l'énonciation qui assigne aux énoncés linguistiques la réalité de leur sémantique en dernière instance.

Quoi qu'il en soit - par opposition à la traductologie descriptive, qui s'en tient à une approche a posteriori du «traduit» (comme on dit un «produit») et en reste à l'étude linguistique de ce que certains se plaisent à appeler un «bi-texte» (To $+\mathrm{Tt})$, c'est-à-dire à l'analyse contrastive de l'original et de sa traduction -, l'approche productive et l'approche inductive en traductologie ont ceci de commun qu'elles s'assignent pour tâche de prendre pour objet la traduction au sens dynamique, le processus de la traduction ou, si l'on veut, le "traduire». Outre la différence déjà notée dans l'échéancier de la recherche, ce qui les distingue, c'est aussi corollairement leurs deux attitudes respectives touchant ledit processus de la traduction. La traductologie productive s'efforce d'anticiper et, donc, de contribuer à débloquer, à "produire» ce processus: en ce sens, elle est une praxéologie, dont la méthodologie s'apparente avec celle d'une recherche-action (voir sup.). Son affaire, c'est le processus de la traduction à venir; alors que la traductologie productive entend procéder à l'étude de ce processus en cours.

Pour être en mesure d'en produire une connaissance scientifique (stricto sensu), cette dernière devra objectiver le phénomène; et, partant, elle se retrouve confrontée aux apories épistémologiques inhérentes aux sciences humaines, dont le manque de place m'interdit de traiter ici (voir Ladmiral 2003: 341-345). Pour l'heure, je voudrais seulement mettre l'accent sur deux ou trois aspects particuliers ou dérivés de cette vaste problématique.

D'abord, il semble qu'il y ait une tendance à la «parcellarisation» des objets de recherche. C'est ce à quoi on assiste en linguistique où, par exemple, les recherches extrêmement "pointues» que mènent des personnalités marquantes comme Pierre Cadiot ou Jean-Jacques Frankel dans le domaine de la sémantique en viennent à mettre en lumière des aspects nouveaux mais excessivement restreints du langage, débouchant sur une formalisation de ces phénomènes que je me hasarderai à qualifier de «micrologique» («microglossique» en l'occurrence). Il n'en va pas autrement de la traductologie inductive. Point n'est besoin, au demeurant, de souligner la pertinence des recherches sémantiques pour la traduction. Cela dit, pour une traductologie inductive ou scientifique, l'essentiel regarde du côté de la psychologie cognitive, autant et plus que du côté de la linguistique. Encore que les recherches de pointe en sciences du langage (en sémantique, par exemple) conduisent ces dernières à rejoindre les sciences cognitives, sinon à s'y intégrer.

Par ailleurs, la neurolinguistique a mis en évidence le fait que la fonction du langage réside en un faisceau de compétences très spécialisées et que l'organisation cérébrale qui en est le support présente un caractère modulaire. - Dans cet esprit général, il est inévitable que la traductologie inductive dégage certaines variables isolées, méthodologiquement artificialisées, au sein des processus en traduction, en y privilégiant souvent l'interprétation par rapport à la traduction (stricto sensu), et qu'elle débouche donc sur des études ponctuelles («micrologiques»). En attendant que vienne le temps de la grande synthèse de tous ces résultats isolés sous les auspices d'une théorie générale dans le cadre des sciences cognitives, mais dans un avenir plus ou moins lointain. Dans ces conditions, il est clair que ces différents acquis de recherche 
(en traductologie inductive comme en sémantique) ne sont pas actuellement opérationnalisables sur le plan de la pratique traduisante et que, donc, on n'ait là encore que la traductologie de demain et même, plus probablement, d'après-demain.

Il convient d'ajouter encore une considération à tout ce qui vient d'être dit. Alors que pendant toute une période initiale du déploiement de la science il a pu sembler que la simplicité d'un modèle ou d'une théorie (son «élégance» mathématique) était un gage de scientificité, il apparaît qu'il n'en est plus vraiment ainsi. C'est pourquoi il n'y a sans doute pas lieu de voir une faiblesse épistémologique dans la «sophistication» des modèles sur lesquels débouchent provisoirement ces recherches: ce n'est pas tant la théorie qui est compliquée que le réel qu'elle prend pour objet qui est complexe. Jointe aux exigences de la méthode expérimentale, cette complexité concourt à faire que ladite parcellarisation tende à être inévitable; et elle contribue aussi à retarder le calendrier de la recherche.

Curieusement, ce qui est vrai des objets de recherche semble l'être aussi des chercheurs eux-mêmes et on dirait que la recherche en traductologie inductive obéit à la même logique de parcellarisation, comme si elle ne pouvait avancer que par àcoups. En sorte qu'au bout du compte on n'aurait encore qu'un archipel de connaissances, mais pas encore un continent de savoir... Parmi les pionniers qui ont commencé à étudier scientifiquement les processus en traduction, sur le plan psycho-cognitif, il convient de citer ce qui s'est fait autour du G.É.L. (Groupe d'Études du Langage) organisant une collaboration interdisciplinaire entre l'É.S.I.T. et la Faculté de médecine de l'Université de Paris-XII-Créteil (voir Barbizet, Pergnier et Seleskovitch 1981). Puis c'est à Genève qu'est allé souffler l'esprit de la traductologie inductive, avec les travaux de Erika Diehl et, plus récemment, de Hannelore LeeJahnke ${ }^{8}$. Entre temps, il y a eu aussi les recherches de Jean-Luc Nespoulous, à l'articulation de la neurolinguistique et de la psycholinguistique, et de bien d'autres (Nespoulous et Leclercq 1990). Très récemment, dans la mouvance de l'Université de Bologne et de son École de traducteurs (SSLiMIT), Michèle Lorgnet est à l'origine d'un projet de publications prometteuses ${ }^{9}$. Cela n'aurait pas de sens de prolonger ici cette liste, au demeurant lacunaire et (involontairement) sélective. Je voulais seulement indiquer le caractère "éclaté» de ces recherches, sinon leur manque de suivi.

Cette situation particulière tient en partie au fait que la recherche en la matière en est encore au stade des commencements et il en va souvent ainsi en histoire des sciences. Il y a aussi les difficultés propres à l'«objet» (humain) étudié, et notamment sa complexité déjà notée. À quoi vient s'ajouter une difficulté spécifique: la traductologie inductive (ou scientifique) se trouve d'emblée confrontée au problème épineux de l'accès aux données. S'agissant de connaître la réalité des processus en traduction tels qu'il se produisent dans le cerveau du traducteur, on va devoir mettre au point différents dispositifs d'investigation qui ne pourront recueillir et interpréter que des données indirectes - sauf à s'imaginer qu'on pourrait ouvrir le crâne d'un traducteur (horresco referens) et qu'on verrait tout! un peu comme dans le conte espagnol où le diable boiteux soulève le toit des maisons pour percer les secrets des hommes... Sauf qu'on n'y verrait rien!

Toujours est-il que ces contraintes sont à l'origine de ce que j'appellerai des cas d' «hybridation épistémologique». C'est ainsi que pour opérationnaliser l'approche inductive, Hannelore Lee-Jahnke est conduite à avoir recours à ce qu'elle appelle joliment «l'introspection à haute voix», comme l'avait fait Hans Peter Krings (voir sup.). 
De même, Michèle Lorgnet prend bien pour objet de connaissance la réalité psycholinguistique des processus en traduction, mais il lui faut en passer par un dispositif d'analyse des données (les énoncés) qui se situe sur le plan linguistique et rhétorique ${ }^{10}$ - Maintenant, si j'en reviens à l'analogie métaphorique du diable boiteux qui vient d'être évoquée et à l'idée d'un accès plus direct aux mécanismes cérébraux qui soustendent les processus en traduction, je dirai qu'on n'a sans doute pas encore assez mis à contribution les moyens récents de l'imagerie médicale pour examiner empiriquement les zones du cerveau qui sont activées pendant qu'un sujet traduit. Comme on sait, c'est ce qu'a fait notamment un Howard Gardner, dans un tout autre domaine, pour en venir à sa théorie des intelligences multiples (voir Gardner 1996).

Quant au concept de déverbalisation qui, encore une fois, relève à mes yeux de la traductologie productive pour laquelle je plaide, il n'y a point d'apparence qu'il dût être validé par une traductologie inductive. La recherche entreprise en ce sens par William P. Isham ne semble pas pouvoir être concluante. Peut-être est-ce un concept trop général, tant il est vrai qu'il est découpé aux mesures du vécu mental, alors que la réalité modulaire du fonctionnement cérébral obéit à une logique spécifique, et différente. Quant au salto mortale, il ressortit à la rhétorique d'une phénoménologie existentielle du processus de la traduction; et si l'on se figurait pouvoir en trouver le répondant dans la réalité objective, ce serait opérer un saut aventuré par rapport aux données de l'expérience - un saut qu'à bon droit on pourra dire épistémologiquement périlleux...

Paris, le lundi $1^{\text {er }}$ novembre 2004, en la fête de la Toussaint.

Nota bene: Compte tenu des limites imparties à la présente étude, il m’a fallu souvent ne faire qu'allusivement référence à certains aspects des problèmes dont j'avais déjà traité ailleurs. C'est pourquoi, conformément à un usage de plus en plus répandu dans les publications en sciences humaines (et qu'on peut trouver agaçant), j'ai dû citer plusieurs de mes propres travaux: la présente étude s'inscrivant, en effet, dans le cadre d'une réflexion d'ensemble, dont c'était l'occasion de faire apparaître la cohérence, et avec laquelle il ne m'a pas semblé inutile de marquer certains points de contact. C'était aussi une façon d'alléger cette même étude qui menaçait de prendre une ampleur excessive. Et puis, je suis quant à moi reconnaissant aux auteurs que je lis quand ils me fournissent des indications de cette nature, qui me permettent d'approfondir tel ou tel point. Enfin, ce m'a été souvent l'occasion de mentionner divers numéros spéciaux de revues et autres publications collectives consacrés aux thèmes abordés, dont le lecteur pourrait n'avoir pas eu connaissance. En revanche, je me suis limité à très peu de choses pour ce qui est des références bibliographiques en général.

\section{NOTES}

1. Voir les trois volumes de l'Hommage à Danica Seleskovitch à paraître très prochainement aux Éditions Minard-Les Belles Lettres (Paris).

2. Voir Ladmiral (1995: 414 sq.). Je ne m’étends pas ici sur cette problématique de la dynamique des groupes bilingues, dont j'ai abondamment traité dans mon livre: Ladmiral et Lipiansky (1995: 2176), voir aussi Ladmiral (1982).

3. Il y aurait là matière à toute une discussion touchant l'épistémologie des sciences humaines à laquelle il ne peut être fait référence qu'allusivement ici et dont j'ai traité ailleurs: Ladmiral 2002a: VI sq.; 2002c: 337 sq. et 2003: 341-345.

4. Ici, comme au demeurant plus haut à plusieurs reprises, je fais référence à la typologie des «âges de la traductologie» que je me suis hasardé à proposer - et que je me suis plu à appeler (cum grano salis) mon quatrain traductologique dans la mesure où les quatre termes que j'ai retenus riment deux 
à deux et forment ce qui s'appelle un double homéotéleute. En l'occurence, j’ai proposé de distinguer quatre modes de discours traductologiques: la traductologie normative ou prescriptive (qu'on pourra dire encore "pré-linguistique») et la traductologie descriptive (qui est d'obédience linguistique, voir sup.), la traductologie scientifique ou inductive et la traductologie productive (Ladmiral 1987 b et 1997).

5. Ce serait là ma façon de «traduire» le concept auquel a recours Heidegger pour thématiser cette problématique: Umsicht - voir Ladmiral 1998: 139 et passim.

6. Au-delà de ce rapprochement, il $\mathrm{y}$ a au demeurant des analogies profondes entre psychanalyse et traductologie que les limites imparties à la présente étude m'interdisent de développer ici: voir notamment Ladmiral 1998: 140 et last but no least Arrivé 1987. Sur l'ensemble de la problématique qui vient d'être abordée très rapidement, voir Ladmiral 1987a: 195 sq.

7. C'est le titre que j'ai donné à mon livre: Ladmiral 2002a - sans revendiquer bien sûr les certitudes de la géométrie pour la théorie traductologique. Mais tel est bien pourtant le sens que prend le concept de théorème en géométrie: c'est une «station» importante dans le déroulement de la démonstration qui se trouve «capitalisée» dans une formulation permettant le réemploi. - D’une façon générale, encore une fois, je dois m'en tenir ici au «sténogamme» de quelques indications allusives, renvoyant pour le reste le lecteur à mes publications antérieures: voir notamment Ladmiral 1997: 36-40 et 2002a: 211-257, etc.

8. Voir Diehl 1983 et Lee-Jahnke 1998; voir aussi la contribution de Hannelore Lee-Jahnke au présent numéro de Meta: «New Cognitive Approaches in Process-oriented Translation Training».

9. Lorgnet 2004 - c'est la première livraison des «Cahiers du R.A.P.T.» (Recherches sur les aspects psycholinguistiques de la traduction); la seconde devrait paraitre dès avant la fin 2004. Je consacrerai une prochaine étude à une critique des deux plaquettes. Quant à la SSLiMIT (Scuola Superiore di Lingue Moderne per Interpreti e Traduttori), elle est sise à Forlì et relève de l'Université de Bologne.

10. Au reste, l'intérêt que porte cet auteur aux ressources de la rhétorique n'est pas nouveau; et ce m'est l'occasion de signaler au passage un de ses ouvrages antérieurs: Lorgnet 1995. Mais il s'agissait alors d'un ouvrage d'ordre didactique.

\section{RÉFÉRENCES}

Arrivé, M. (1987): Linguistique et psychanalyse. Freud, Saussure, Hjelmslev, Lacan et les autres, préf. Jean-Claude Coquet, Paris, Méridiens/Klincksieck.

Bailly, D. (1984) : Éléments de Didactique des langues, L'activité conceptuelle en classe d'anglais, 2 vol., Lyon, Les Langues modernes.

Barbizet J., Pergnier, M., D. Seleskovitch, Danica (éds.) (1981): Comprendre le langage. Le point de vue des linguistes. Le point de vue des traducteurs interprètes. Le point de vue des neuropsychologues (Actes du colloque de Créteil : 25-27 septembre 1980), Paris, Didier Érudition (collection «Linguistique», $\mathrm{n}^{\circ} 12$ ).

Cadiot, P. et Y.-M. Visetti (2001): Pour une théorie des formes sémantiques. Motifs, profils, thèmes, Paris, P.U.F. (coll. «Formes sémiotiques»).

Dienl, E. (1983) : «Psycholinguistik und Dolmetscherpraxis - ein Versuch der Vermittlung», Translation Theory and Its Implementation in the Teaching of Translating and Interpreting, Tübingen, G. Narr Vlg. (Tübinger Beiträge zur Linguistik, nº 247), p. 231-240.

Gardner, H. (1996): Les Intelligences multiples, trad. fr. Ph. Evans-Clark, M. Muracciole et N. Weinwurzel, Paris, Retz (coll. «Psychologie»).

Hewson, L. et J. Martin (1999): Redefining Translation. The Variational Approach, London and New York, Routledge.

KaYser, W. (1948): Das sprachliche Kunstwerk, Eine Einführung in die Literaturwissenschaft, Bern, Francke.

Krings, H. P. (1986): Was in den Köpfen von Übersetzern vorgeht, Tübingen, G. Narr Vlg. (Tübinger Beiträge zur Linguistik, $\mathrm{n}^{\circ} 291$ ).

Ladmiral, J.-R. (1971): «Le discours scientifique», Revue d'Ethnopsychologie, t. XXVI/nos 2-3, septembre, p. 153-191.

Ladmiral, J.-R. (1982) : «Traduction et psychosociologie», Meta 27-2, Les Presses de l'Université de Montréal, p. 196-206. 
Ladmiral, J.-R. (1986) : «Sourciers et ciblistes», Revue d'esthétique 12, p. 33-42.

Ladmiral, J.-R. (1987a): «Technique et esthétique de la traduction», Actes des Journées européennes de la traduction professionnelle (UNESCO, Paris 25-26 mars 1987): Encrages (Hachette et Université de Paris-VIII: Vincennes à Saint-Denis), nº 17, Printemps 1987, p. 190-197.

Ladmiral, J.-R. (1987b): «Traductologiques», Retour à la traduction = numéro spécial de la revue Le Français dans le monde (Recherches et applications), éd. Marie-José CAPELLE/Francis Debyser/Jean-Luc Goester, août-septembre 1987, p. 18-25.

Ladmiral, J.-R. (1995): «Traduire, c'est-à-dire... - Phénoménologie d'un concept pluriel», Meta 40-3, septembre 1995, p. 409-420.

Ladmiral, J.-R. (1997) : «Les quatre âges de la traductologie - Réflexions sur une diachronie de la théorie de la traduction ", L'histoire et les théories de la traduction, Les actes (colloque de Genève: 3-5 octobre 1996), Berne et Genève, ASTTI \& ÉTI, p. 11-42.

Ladmiral, J.-R. (1998) : «Théorie de la traduction: la question du littéralisme», Transversalités. Revue de l'Institut catholique de Paris 65, janvier-mars 1998, p. 137-157.

Ladmiral, J.-R. (2001): "Métaphraséologiques", The Contribution of Language Teaching and Learning to the Promotion of a Peace Culture (Actes du colloque A.I.L.A. de Thessalonique: 9-12 décembre 1999), Université Aristote de Thessalonique, p. 97-117.

Ladmiral, J.-R. (2002a): Traduire: théorèmes pour la traduction, Paris Gallimard, 2002 («Tel», $\left.\mathrm{n}^{\circ} 246\right)$, rééd. augmentée d'une préface.

LADMiral, J.-R. (2002b) : «La traduction, un concept aporétique?», Identité, altérité, équivalence? la traduction comme relation. Actes du colloque international tenu à l'ÉSIT les 24, 25 et 26 mai 2000 en hommage à Marianne LEDERER, textes réunis et présentés par Fortunato IsRAËL, Paris-Caen, Lettres modernes/Minard (coll. «Cahiers Champollion», $n^{\circ}$ 5), p. 117-144.

Ladmiral, J.-R. (2002c) : «De la linguistique à la littérature: la traduction», Le Signe et la lettre, Hommage à Michel Arrivé, textes réunis par J. Anis, A. EskénAZi et J.-F. JeAndillou, Paris, L’Harmattan (coll. «sémantiques», sous la dir. de Marc Arabyan), p. 337-347.

LADMiRAL, J.-R. (2003) : «La traductologie au XXI e siècle: de la linguistique à la psychologie», Traduire au XXI siècle: tendances et perspectives. Actes du colloque international de Thessalonique: 27-29 septembre 2002, éd. Tonia Nenopoulou, Faculté des Lettres de l'Université Aristote de Thessalonique, p. 336-346.

LADMiRAL, J.-R. (2004): «Dichotomies traductologiques », La Linguistique, volume 40, fasicule 1, p. 25-49. Il s'agit en l'occurrence d'un numéro thématique entièrement consacré à la traduction, sous la direction de Claude Tatilon.

Ladmiral, J.-R. et E. M. Lipiansky (1995): La communication interculturelle, Paris, Armand Colin (Bibliothèque européenne des sciences de l'éducation).

Ladmiral, J.-R. et H. Meschonnic (sous la dir. de) (1981) : La traduction, numéro spécial de la revue Langue française, ${ }^{\circ}$ 51, septembre 1981.

Lee-Jahnke, H. (1998) : «L'introspection à haute voix: recherche appliquée», Enseignement de la traduction et la traduction dans l'enseignement, sous la dir. de Jean Delisle et Hannelore LeeJahnKe, Les Presses de l'Université d'Ottawa (coll. «Regards sur la traduction»), p. 155-183.

Lorgnet, M. A. (1995): Pour une traduction holistique - recueil d'exemples pour l'analyse et la traduction, Bologne, Cooperativa Libraria Universitaria Editrice Bologna (Biblioteca della Scuola Superiore di Lingue Moderne per Interpreti e Traduttori, Forlì).

Lorgnet, M. (2004): Le traducteur et ses mémoires, Turin/Paris, L'Harmattan (Cahiers du R.A.P.T., $\left.n^{\circ} 1\right)$.

Lourau, R. (1978): Sociologue à plein temps, Paris, L'Épi.

Nespoulous, J.-L. et M. LeClercQ (éds) (1990): Linguistique et neuropsycholinguistique: tendances actuelles, Paris, Éditions de la Société de Neuropsychologie de langue française.

Seleskovitch, D. et M. Lederer (1984): Interpréter pour traduire, Paris, Didier Érudition (coll. «Traductologie», $\mathrm{n}^{\circ} 1$ ).

SKInNER, B. F. (1957): Verbal Behavior, New York: Appleton-Century-Crofts. 